\title{
Strates
}

STRATES Matériaux pour la recherche en sciences sociales

14 | 2008

Espaces du quotidien

\section{Point de méthode : géographie des territoires et territorialités à Damas (Syrie)}

\section{Stéphane Schehl}

\section{(2) OpenEdition \\ 1 Journals}

\section{Édition électronique}

URL : http://journals.openedition.org/strates/6745

DOI : $10.4000 /$ strates. 6745

ISSN : $1777-5442$

\section{Éditeur}

Laboratoire Ladyss

\section{Édition imprimée}

Date de publication : 1 janvier 2008

Pagination : 165-176

ISBN : 0768-8067

ISSN : 0768-8067

\section{Référence électronique}

Stéphane Schehl, «Point de méthode : géographie des territoires et territorialités à Damas (Syrie) », Strates [En ligne], 14 | 2008, mis en ligne le 13 mars 2013, consulté le 08 septembre 2020. URL : http:// journals.openedition.org/strates/6745; DOI : https://doi.org/10.4000/strates.6745

Ce document a été généré automatiquement le 8 septembre 2020.

Tous droits réservés 


\title{
Point de méthode : géographie des territoires et territorialités à Damas (Syrie)
}

\author{
Stéphane Schehl
}

1 L'analyse de la mobilité ordinaire à Damas permet de compléter utilement l'approche par les paysages et les attributs matériels de la cité (Schehl, 2006). La mobilité, caractérisée par des déplacements physiques réguliers effectués au sein de l'agglomération de Damas produit un nouveau mode de territorialisation qui articule modernité et tradition sociale et familiale arabe qui renouvellant l'organisation de l'espace urbain. Il s'agit ici de voir en quoi des pratiques ordinaires en situation de mobilité, les réseaux d'hommes et l'emploi informel permettent de nuancer le rôle décisif des clivages religieux et ethniques dans la structure sociale.

2 L'absence de travaux sur le vécu et l'ordinaire dans la littérature francophone sur Damas (Dupret et Chiffoleau, 2005) m'a conduit à privilégier l'échelle de l'individu pour appréhender des pratiques, des usages qui participent du vécu ordinaire des personnes qui habitent, parcourent, utilisent la ville et dessinent un réseau de lieux hors de toute logique de continuité physique. La pluralité des territoires ordinaires observés est liée aux diverses formes d'appartenances, d'allégeances, de positions sociales et ethniques. Comme dans d'autres pays arabes les allégeances au père, aux personnes plus âgées, aux Zaîm (chefs) continuent à structurer les formes de relation à autrui. L'observation d'individus fréquentant toutefois les mêmes lieux permet de reconsidérer la sociabilité, le rôle des réseaux d'hommes ainsi que l'importance des compétences individuelles et collectives dans la constitution des territoires de l'ordinaire. Les échanges au sein de réseaux réticulaires à plus petite échelle permettent de saisir la spatialité complexe de ceux qui pratiquent la ville au quotidien.

3 Au-delà des flux statistiques, ce sont les territoires et les pratiques individuelles qui importent: ils permettent de cerner des processus de différenciations sociales et territoriales. 


\section{Les pratiques ordinaires en situation de mobilité}

4 Sur un échantillon de population de Wadi al Kâhf, j'ai retracé les espaces fréquentés et traversés de manière ordinaire par ses habitants (carte 1). Il s'agit d'un quartier d'habitat informel récent de Damas situé sur le Mont Qasioun. L'échantillon obtenu par grappe représente 70 individus sur 20 familles ayant leur résidence habituelle à Wadi al Kâhf. Ce sont majoritairement des descendants d'immigrés (Tcherkesses, Turcs, Séoudiens) ou de réfugiés (Syriens du Golan, Palestiniens, Libanais originaires du Sud Liban et de la Beqaa).

Carte 1. Quartiers fréquentés de manière ordinaire par les habitants de Wadi al Kâhf.

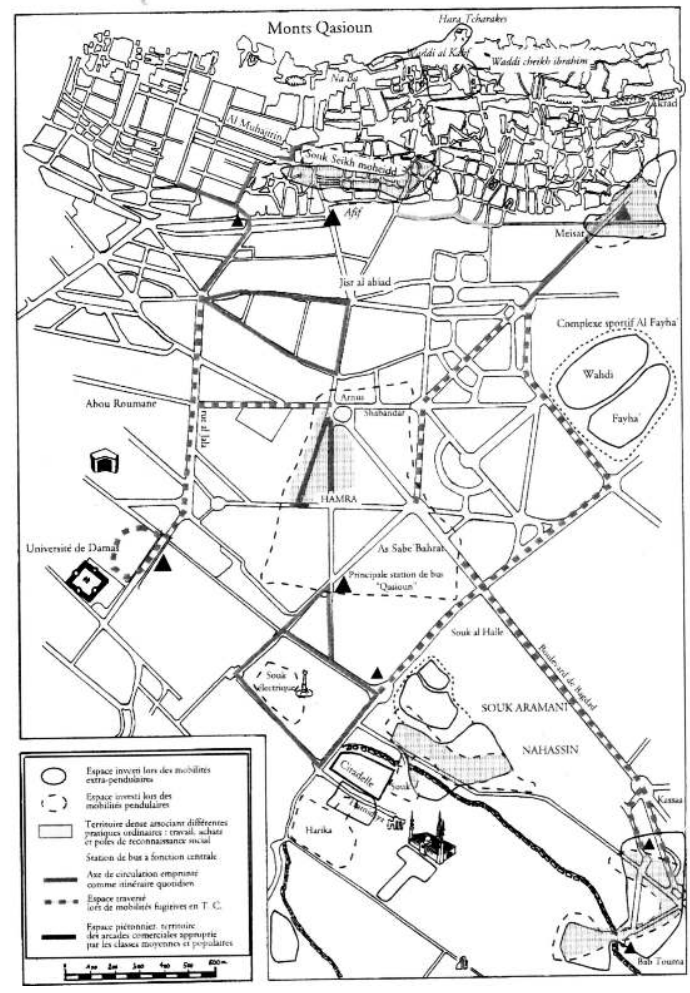

5 Les mobilités liées ou non au travail et à la scolarité ainsi que les mobilités hors travail ont retenu mon attention. Les interactions ordinaires hors des lieux de résidence et de travail, la fréquentation des points de rupture de charge comme les gares, les lieux de transit, les stations de bus, ce qu'Isaac Joseph (2004) appelle les «lieux mouvement », mais aussi la fréquentation de lieux d'approvisionnement et de détente, permettent de retracer toute une géographie des lieux de liens faibles et éphémères, pratiquées de manière répétitive suivant une fréquence pluri-hebdomadaire.

On repère aussi hors du quartier habité, des lieux de brassage de population où s'expériemntent d'autres formes de relations: pratiques de consommation des souks, lieux de détente, de loisir, de rencontre et de visite. Grâce à une enquête utilisant une technique "libre et spontanée » pour reprendre la formule de Cauvin (1999), nous remarquons que ces lieux se caractérisent par un paysage, des usages et des formes d'échanges très nombreuses et qui structurent beaucoup la territorialité. 
Interactions au souk al Atik près de la place Merjé.



\section{Les réseaux d'hommes et l'emploi informel : l'épine dorsale des territoires éclatés}

7 L'observation directe permet de déterminer qu'il existe des lieux de passage quasi incontournables comme les grandes stations de bus. Les « lieux mouvement » comme la station de bus de jisr-al-Raîs sont des lieux de brassage de population, de rencontre et d'échange marchand, ils sont le support de nombreux petits métiers de rue (Hirafiyyîn), vendeurs à la sauvette, vendeurs de cassettes audio mais aussi d'aliments et de boissons à emporter. Les populations flottantes, comme les vendeurs de billets de loterie, de fruits empiètent sur l'espace public et créent des groupes informels qui se recomposent au gré des flux de voyageurs. Tout au long de la journée se succèdent aux abords des quais divers types de commerces de rue. Cette gare routière sous un pont est la plus importante avec celle de Garage Baramké située non loin, à proximité de l'Université.

Nous avons regroupé les lieux cités par les répondants à notre enquête au sein de territoires identifiés comme pertinents à l'échelle du vécu intra-urbain des habitants du quartier. Ces informations (lieux, données sociales, pratiques) permettent de réaliser une base de données qui croise les lieux fréquentés, les caractéristiques humaines, sociales et économiques des répondants. Il est alors possible de déceler l'importance des classes d'âges, des positions dans le cycle de vie et des relations avec le chef de famille et du genre des individus concernés, l'appartenance à une communauté et à un secteur d'activité. Dès lors, apparaissent des sous-groupes de population croisant le genre, l'appartenance à une classe d'âge ainsi que le statut d'activité. Des étudiants, des femmes actives, des retraités et des jeunes actifs qui cumulent plusieurs emplois et fréquentent de nombreux quartiers en périphérie présentent des similitudes en termes de script de vie et de lieux fréquentés. Les lieux fréquentés et l'agencement spatiotemporel des activités créent des formes de territorialité comparables pour des 
individus partageant les mêmes contraintes familiales, sociales et culturelles. Les entrepreneurs décrivent des trajectoires liées aux besoins de leur activité et aux chantiers qu'ils suivent. Leur perception et leur connaissance de la ville donnent lieu à une géographie des lieux utiles au quotidien. C'est par exemple le cas du «travail libre » des conducteurs de Suzukis qui ont répondu à nos questions.

Leurs cartes mentales (cartes 2 et 3) proposent une représentation de leur espace vécu et perçu lié à leur pratique quotidienne. Ils soulignent les différentes ambiances, la présence de certains groupes ethniques ou religieux surreprésentés dans certains îlots (Chi'ites, Caucasiens Abaza...), mais aussi la qualité de la voie et la présence d'autres modes de transport.

Le facteur le plus discriminant semble être l'âge qui détermine des contraintes et un potentiel de mobilité en fonction des positions dans le cycle de vie, des scénarios et des formes de potentiels assez tranchés entre individus appartenant au même quartier et de même sexe. Les entrevues et les cartes mentales des plus jeunes permettent d'identifier des secteurs de la ville et des activités propres aux jeunes (lieux de réunion, restaurants, snacks, mais aussi des salles de sport et la fréquentation d'établissements scolaires). Les jeunes gens ne fréquentent pas les mêmes lieux en fonction de leur âge, de leur sexe et de leur niveau d'étude. Certains cafés et clubs ont une clientèle bien définie en terme d'âge et de revenu.

L'analyse des mobilités par genres et par classes d'âges va au-delà des catégories socioprofessionnelles, des ethnies et des groupes religieux habituellement invoqués. Les convergences, les échanges et regroupements permettent de définir de nouvelles centralités et temporalités liées à des profils (femmes divorcées, entrepreneurs, jeunes gens...). Certaines pratiques sont réservées aux familles, aux couples.

Carte 2. Carte mentale des territoires ordinaires d'un jeune habitant du quartier de Wadi al Kâhf.




Carte 3. Carte mentale des territoires ordinaires d'un conducteur de taxi collectif « Suzuki ».

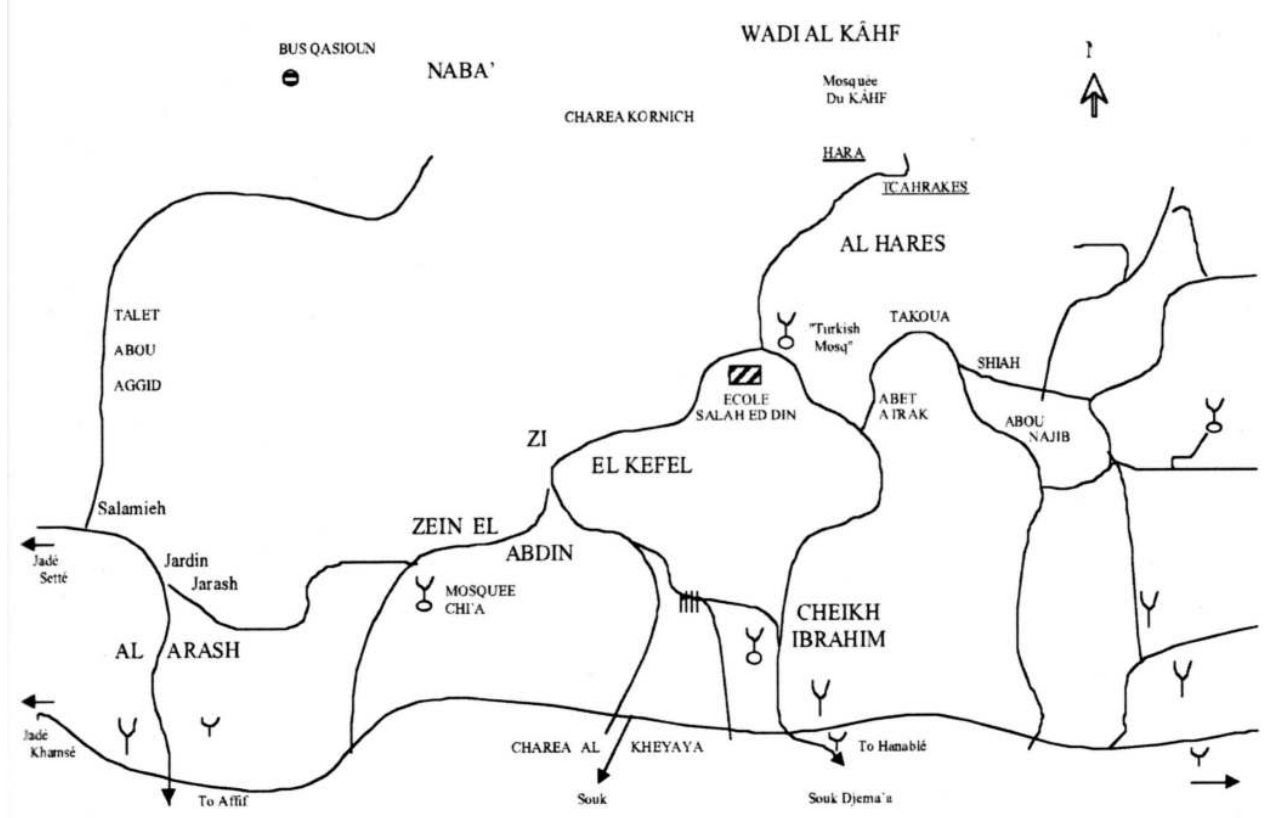

La diversité des formes de socialisation permet de retracer des profils très divers. Les jeunes femmes par exemple n'ont absolument pas les mêmes pratiques avant et après leur mariage, la décohabitation engendre souvent un élargissement des lieux fréquentés. L'accès à un emploi favorise l'accès à des loisirs et à des pratiques sportives et culturelles dans une moindre mesure souvent interdites aux plus jeunes. Il en est de même pour les habitants du Kâhf peu aisés mais disposant de « connaissances » qui leur permettent d'accéder à des lieux qui leur sont habituellement interdits du fait du coût, des barrières psychologiques ou encore de barrières physiques. Le fait d'être invité et de connaître des membres d'un groupe permet d'accéder à de nombreux espaces privés inaccessibles sans avoir recours à un intermédiaire (cas des clubs).

Les élèves du secondaire expliquent, par exemple, les choix entre proximité et éloignement qui motivent la sélection de certains lieux d'enseignement, de consommation et de pratique sportive.

L'informel occupe une part importante dans la vie ordinaire des habitants. Leur âge, leur genre ainsi que leur appartenance ethnique et socioprofessionnelle conditionnent leur aptitude à la mobilité, la teneur des interactions et l'accès aux divers espaces publics. Certaines interactions démontrent que l'espace public met en situation de coprésence de véritables échanges entre divers groupes.

15 L'accès à des formes de capital social, culturel et économique discrimine aussi les individus qui fréquentent ou non différents établissements comme des lieux d'échange et de rencontres. Les plus aisés pratiquent ainsi des lieux de loisir et de détente qui sélectionnent financièrement les clients. Les habitants du Kâhf n'accèdent que très rarement aux clubs, restaurants et cafés pratiquant des tarifs exorbitants. Concernant les pratiques de location de fermes pour le week-end, les jeunes du Qasioun sont exclus de ce type de mode de détente.

Une analyse des réseaux d'hommes permet de mieux appréhender la spatialité des réseaux de territoires qui contredisent l'idée d'enclavement et de repli. De nombreux groupes informels se créent à l'aune des échanges et des déplacements à des moments 
et en des lieux bien précis. Ils sont organisés autour d'individus disposant d'un statut particulier au sein du quartier mais aussi dans leurs relations avec d'autres hommes en d'autres lieux, ils sont multi-appartenants. Ils cumulent plusieurs répertoires d'identité et peuvent ainsi naviguer plus aisément dans plusieurs milieux.

17 Certains habitants du Kâhf fréquentent, par exemple, des milieux plus aisés ou des personnes bien introduites dans l'appareil bureaucratique. Ils disposent ainsi d'appuis politiques (notamment au sein du parti Baath).

18 L'aptitude à la mobilité et une bonne connaissance des ressources des territoires sont des atouts primordiaux qui permettent de s'intégrer et de créer de la richesse. Les Mou'alem $^{1} \mathrm{du}$ quartier redistribuent ainsi des emplois (en recrutant par cooptation) et partagent leur capital relationnel avec les membres de leur réseau, voisins et partenaires. Ils articulent diverses compétences (financières, appuis administratifs, main-d'œuvre) qui sont localisées dans de nombreux quartiers de Damas mais aussi hors de l'agglomération. Cette ouverture sur les autres et les territoires de la ville permet une diffusion des pratiques de rencontre et d'échange entre les membres de ces réseaux.

19 Les territoires animés par des figures du quartier de Wadi al Kâhf, les Mou'alem, cumulent plusieurs rôles. Ils apparaissent comme des lieux de pouvoir, de transaction, d'échange de biens et d'informations, de Wastas (pistons) (Bonerandi, 2004), mais aussi comme des lieux de détente et de reconnaissance sociale. Le cumul de divers rôles et l'opportunisme caractérisent les acteurs économiques qui multiplient ainsi les sources de revenu. Chaque vécu, chaque histoire personnelle et familiale, voire le contexte géopolitique, explique en partie leur comportement et leur manière d'appréhender la ville au quotidien. Ce sont des pistes pour mieux comprendre comment ils se sont fixés, comment leur territorialité ordinaire s'est édifiée autour de réseaux d'hommes. Ils sont membres des familles et clans appartenant aux diverses vagues migratoires qui ont successivement urbanisé le nord de Damas depuis plusieurs siècles. Certaines familles d'origine turque tirent de leur patrimoine immobilier des rentes immobilières importantes qui sont réinvesties dans des boutiques situées ailleurs en banlieue. 




Les diasporas semblent jouer un rôle important dans le fonctionnement urbain du fait de la spécialisation de certains réseaux. Dans le bâtiment et les travaux publics (Palestiniens, Syriens originaires du Golan), les transports (Tcherkesses), l'usinage, la peinture sur soie (Turcs), la famille et l'origine géographique jouent un rôle important mais aussi la communauté de destin entre réfugiés (Palestiniens, Libanais originaires de Baalbek, réfugiés du Golan). Certaines diasporas sont concurrentes (Palestiniens, Djoulani, Tcherkesses), d'autres complémentaires en termes de compétences et de formes de capital disponible. Les partenariats entre différents individus peuvent dépasser les clivages ethniques et religieux comme dans les échanges de capitaux et de services: les partenariats dans le secteur du bâtiment et des travaux publics entre musulmans (qui jouent le rôle de Big man comme ensemblier) et chrétiens (Rich man) sont fréquents et permettent de financer des chantiers de second œuvre qui pourvoient de nombreux emplois locaux au sein du Mont Qasioun.

\section{Dépasser les clivages religieux et ethniques}

21 On accède à une autre image de cet espace urbain dilaté, fragmenté mais cohérent en termes de réseaux d'hommes et de territoires complémentaires. On parvient à relativiser l'importance des aspects morphologiques, résidentiels (exclusion, ségrégation résidentielle) et du fait religieux. Les espaces pratiqués au quotidien par les intermédiaires et les personnes bénéficiant d'un emploi dépassent largement le spectre du local et de la proximité immédiate du logement au sein d'un quartier. En fait, les actifs travaillant au sein de ce quartier sont rares à l'exception d'artisans. La majorité des actifs pratiquent de nombreux quartiers pour s'approvisionner, livrer, réparer ou encore accomplir des tâches à domicile. Ils sont très mobiles tout au long de la journée, 
leurs chainnages d'activités sont complexes et ordonnés en fonction de priorités individuelles, professionnelles et familiales objectivables (en fonction des contraintes et des positions familiales de chacun au sein du groupe qui légitiment des obligations).

Les divers degrés d'implication des répondants à l'enquête permettent de comprendre l'importance des pratiques sociales hors de la sphère locale et familiale, sphères qui interagissent beaucoup avec l'économique. Les formes de repli sont extrêmement rares. Les habitants multiplient les formes de lien occasionnant des mobilités hors du quartier.

En somme, la géographie du «travail libre» et des petits métiers contredit toute lecture sociospatiale de quartiers présumés homogènes et antagonistes car les réseaux font apparaître une articulation entre individus appartenant à des confessions ou à des communautés différentes, mais détenteurs de formes de capital et de ressources complémentaires (appareil de production, main-d'œuvre, argent, savoir faire, réseau relationnel, Wasita). Les formalités administratives fort complexes en Syrie profitent à des acteurs intermédiaires pouvant monnayer des "pistons" pour obtenir des documents en matière d'importation, d'urbanisme, de marchés publics...

Nous n'avons pas identifié de fonctionnement exclusif mais des filières marquées par des surreprésentations largement liées à des effets d'hystérésis ${ }^{2}$. Ainsi des territorialités apparaissent différentes de celles évoquées par les orientalistes insistant sur le fait religieux et sa mosaïque levantine. Des formes d'allégeances à des registres traditionnels existent certes (communauté, famille, couche sociale), mais il existe aussi des formes plus modernes comme des liens spécifiques liés à la recherche soit de réseaux personnels soit de partenaires. Les acteurs locaux identifiés sont capables de mixité car ils cultivent des affinités hors des groupes primaires.

Les articulations transnationales et les partenariats entre chrétiens et musulmans démontrent une logique dépassant celle des clivages communautaires classiques souvent mis en avant dans l'analyse des villes arabes. Il en est ainsi d'un Mou'alem sunnite originaire de Kuneitra qui rassemble des fonds prêtés par des familles chrétiennes de Damas pour réaliser des travaux en employant des tâcherons chi'ites. Ce mou'alem et son partenaire chrétien importent des matériaux d'Italie, de France avec l'aide de leurs amis qui financent et payent des "pots de vin » ou les aident à obtenir l'attribution des marchés publics locaux de travaux de second œuvre.

Ceux qui forment des réseaux de fabrication, de transport et de vente de produits licites mais en économie informelle, créent des lieux de négociation de produits chers ou qui font défaut. Ils disposent de possibilités relationnelles qui les aident à circuler et à faire circuler biens et main-d'œuvre. Ces réseaux de commerce ont des antennes transnationales notamment au Liban.

Si des communautés existent bien, elles ne déterminent pas la totalité des opportunités et des interactions individuelles. La mobilicité (Bonerandi, 2004) et le potentiel relationnel de certains individus est très important et assez étendu tant au plan social que spatial (mobilités interurbaines ordinaires et parfois transnationales). 


\section{D'autres formes de socialisation et des territoires éphémères}

D'autres formes de socialisation et de pratiques spatiales amènent à identifier des facteurs discriminants (classes d'âges, classes sexuelles, statut d'emploi, appartenance aux catégories défavorisées...) Une analyse des données permet d'objectiver ces facteurs qui différencient les déplacements (territoires des genres, des couches sociales, des classes d'âges) et leur ampleur (distance temps, distance absolue, recours à divers modes de transport en commun).

Le genre semble être le facteur le plus important après l'âge et la position dans le cycle de vie. Les femmes (y-c. des femmes chef de famille plus âgées) n'occupent jamais le rôle de Mou'alem, d'intermédiaire. Elles occupent souvent des emplois de bureau dans des administrations, de banques et de cabinets d'avocats. Leur profil professionnel est totalement différent de celui des hommes. Elles ont des territorialités spécifiques qui ne recoupent absolument pas celles des hommes du quartier. Ces derniers exercent des emplois dans d'autres secteurs d'activité en d'autres lieux (chantiers, métiers de livreur, chauffeurs). Loin d'être des marginaux, ces actifs issus des couches urbaines moyennes occupent une position centrale dans l'économie syrienne. Les habitants des quartiers périphériques du nord de Damas sont habituellement considérés comme des gens en marge du fait de l'aspect inachevé des habitations (Moukhalafats de Qasioun), de la mauvaise réputation et de leurs mœurs souvent décriés. Il s'agit d'intermédiaires qui arrangent les situations complexes au moyen de leurs connaissances et de leur réseau de relation politique, économique et social. Leurs réseaux réempruntent des réseaux structurants traditionnels sans pour autant s'y limiter strictement.

Les mobilités hors travail engendrent d'autres formes de spatialité, d'autres univers. Certains lieux de loisir et de détente sont réservés aux familles, d'autres aux couples, certains clubs sportifs ne sont pas mixtes comme les piscines; il en est de même pour certains lieux d'approvisionnement, de fast-food et cafés traditionnels aux clientèles bien définies. L'âge et la position dans le cycle de vie créent des ségrégations au sein des lieux de détente et de consommation (clubs, boîtes réservées aux jeunes élites). L'enrichissement d'une minorité citadine à Damas est très visible, (Picard, 2005). Elle tranche avec l'érosion du pouvoir d'achat des retraités et des petits fonctionnaires précarisés qui sont souvent obligés d'avoir recours à d'autres formes d'emploi et de détente (pratique sportive, fréquentation d'un café, d'un club, d'une salle de boxe ou de billard...). Ces pratiques concentrées en un petit nombre de lieux créent des territoires vers lesquels certains individus convergent. Concrètement il s'agit des lieux d'échange, des lieux d'animation comme le souk Sheikh Moheiddin, de Hamra-Salhia (magasins franchisés) et des cafés et des snacks. Les consommateurs présentent en ces lieux des profils socio-économiques et familiaux assez similaires.

31 Les pratiques d'achat sont liées au niveau de revenu disponible; elles opposent les membres de la tabqa jadida (la nouvelle classe aisée) aux habitants des quartiers populaires. Les moins aisés (petits fonctionnaires, tâcherons, journaliers) ont recours à des marchés de substitution alimentés par la contrebande (alimentaire, chaussures, vêtements...) et l'informel (constatations qui se confirment à l'échelle nationale au regard du rapport récent (Meier, 2005) produit pour le PNUD). Les divers lieux d'approvisionnements permettent de définir des marchés et des univers assez fragmentés, les frontières des territoires individuels restant liées aux conditions 
individuelles d'accès à l'urbanité. À Damas, il est possible d'acheter des contrefaçons textiles aux abords de la préfecture et de jisr Victoria. Le soir venu, dans le Souk Hamidiyya à proximité de la Grande Mosquée des hommes proposent des fruits secs de contrebande en provenance du Liban et dans les arrières boutiques de certains commerces se vendent sous le manteau des cigarettes de contrebande provenant de Chypre.

\section{Conclusion}

Tant en termes de mobilité que de pratiques, les territoires et les territorialités des habitants de Damas ne peuvent se résumer à des clivages simples et absolus. Le relationnel joue un rôle prépondérant dans l'édification d'un réseau territorial et social très personnel. La territorialité individuelle emprunte beaucoup les chemins de ces relations personnelles qui structurent l'économie informelle. Les explications ethniques et religieuses des comportements s'estompent au profit de modes de faire de plus en plus liés à des positions socio-économiques (revenus, insertion dans des réseaux économiques, sociaux, ancienneté en ville, forme de capital, lieu de scolarisation des enfants) et familiales qui conditionnent les aires de mobilité. L'informel semble être un moyen de lire le quotidien de la majorité des Syriens qui vivent dans des conditions précaires. Le cumul d'emploi est devenu la règle pour la majorité des couches moyennes dont les fonctionnaires: de nombreux universitaires conduisent un taxi après leurs cours, d'autres sont guides pour des sociétés de tourisme ou donnent des cours d'anglais dans des structures étrangères, des employés d'État cumulent des jobs de plombier, de dépanneurs à domicile. Leurs territoires ordinaires sont ceux de groupes néotraditionnels qui réempruntent des formes anciennes de lien et de réseaux territoriaux qui dépassent largement les limites de l'agglomération de Damas. Ces pratiques banalisées confèrent de la valeur et du sens aux territoires. En fait, il existe une très grande diversité des formes de territorialité au sein d'un même quartier et même au sein d'une même famille élargie. Le grand panel des formes de participation sociale et des formes de développement des réseaux humains et de territoires créent une ville en miettes pour toutes les activités ordinaires individuelles, en somme, une forme de ville à la carte.

\section{BIBLIOGRAPHIE}

Bonerandi E., 2004, « De la mobilité en géographie », Geoconfluences, 5 p., http :www.enslsh.frgeoconfluence/doc/transv/Mobil/

MobilScient.htm $+g$ \%C3A9geographie+de+la+mobilit \%C3 \%A9\&hl =fr. (URL consultée le 19/07/2005).

Cauvin C., 1999, "Propositions pour une approche de la cognition spatiale intra-urbaine ", Cybergéo, $\mathrm{n}^{\circ}$ 72, 26 p., p. 15. 
Dupret B., Chiffoleau S., 2005, La Syrie au présent, IFPO-CNRS,

http :www.ifporient.orgrubrique.php3 ?id_rubrique =59 (URL consultée le 09/03/2006).

Godelier M., 1991, Big Men and Great Men : Personifications of Power in Melanesia, Cambridge University Press-Maison des Sciences de l'Homme, 320 p.

Joseph I., 2004, Goffmann et l'Écologie urbaine, Annales de la Recherche Urbaine, nº 95, juin 2004, p. 130-134.

Meier D., 2005, « Y a-t-il une classe moyenne au Liban? », Carnets de bord en sciences humaines, $\mathrm{n}^{\circ}$ 10, déc. 2005, p. 46-54.

Picard E., 2005, « Syrie : la coalition autoritaire fait de la résistance », Politique étrangère, 4/2005, p. 757-768.

PNUD, 2005, Poverty in Syria : 1996-2004, rapport, Programme des Nations unies pour le développement, New-York, $134 \mathrm{p}$.

Schehl S., 2006, Territoires, territorialité et mobilité ordinaire à Damas (Syrie), Thèse de Géographie, École doctorale SHS, Université de Tours, $681 \mathrm{p}$.

Tarrius A., Bernet O., 2003, « Migrants transnationaux, "fourmis" de la mondialisation », communication lors d'un séminaire de formation, IUFM d'Aix-Marseille, $12 \mathrm{p}$.

Tarrius A., Misaoui L., 2000, Les fluidités de l'ethnicité : réseaux de l'économie souterraine, rapport pour la direction du patrimoine, ministère de la Culture et PUCA-METL, Université du Mirail, Toulouse, $147 \mathrm{p}$.

\section{NOTES}

1. Les Mou'alem sont des figures locales, patrons, notables; ils occupent une position centrale dans la société syrienne. Sorte de chef nous l'assimilons au «big man» décrit par Maurice Godelier (1991).

2. L'hystérésis est la présence d'un effet retard observé dans un phénomène par rapport à sa cause. L'effet persiste alors que la cause physique générant le phénomène n'existe plus.

\section{RÉSUMÉS}

L'analyse de la mobilité ordinaire à Damas menée à partir des réseaux sociaux et de l'emploi informel permet de mieux comprendre l'organisation des territoires de l'ordinaire. Sur un échantillon de population de Wadi al Kâhf, nous avons retracé les pratiques et les espaces fréquentés au quotidien par ses habitants. Au-delà de la comptabilisation des flux, ce sont les territoires et les pratiques individuelles qui importent. L'observation révèle les pratiques et les lieux liés aux profils sociaux, économiques et culturels. L'appartenance à une communauté et à un secteur d'activité conditionne également les formes de territorialité. Une analyse statistique des données permet d'objectiver les résultats issus des enquêtes individuelles. Les explications ethniques et religieuses des comportements s'estompent au profit de modes de faire de plus en plus liés à des positions socio-économiques individuelles et familiales qui conditionnent les aires 
de mobilité. L'informel nous semble être un moyen de lire le quotidien de la majorité des Syriens qui vivent dans des conditions précaires.

The analysis of ordinary mobility that was carried out in Damas on the basis of human networks and informal employment enables a better understanding of how ordinary territories are organized. We traced the inhabitants' practices and the spaces they use on a daily basis from a sample of the population of Wadi al Kâhf. Beyond statistical noise, what matters most are territories and individual practices. Our observation reveals the practices and places that are related to social, economic and cultural profiles. Belonging to a community and a particular activity sector also affects the various forms of territoriality. A data analysis enables obtaining objective results stemming from individual surveys. Behaviours' ethnical and religious explanations fade away in favour of ways of doing that are more and more related to individual and family socio-economic positions which affect mobility areas. What is informal seems to be a way of reading the daily lives of most Syrian people who live in precarious conditions.

\section{INDEX}

Mots-clés : quotidien, mobilités, territoire, transports, sociabilité, identités

Index géographique : Syrie

\section{AUTEUR}

\section{STÉPHANE SCHEHL}

Docteur en géographie de l'université de Tours, Responsable des études, DDE 91, Ministère de l'Aménagement stephane.schehl@equipement.gouv.fr 